\title{
Very Weak Signals (VWS) detected by stacking method according to different astronomical periodicities (HiCum)
}

\author{
M. van Ruymbeke ${ }^{1}$, P. Zhu ${ }^{1}$, N. Cadicheanu ${ }^{2}$, and S. Naslin ${ }^{1}$ \\ ${ }^{1}$ Royal Observatory of Belgium, Belgium \\ ${ }^{2}$ Institute of Geodynamics of Romanian Academy, Bucharest, Romania
}

Received: 20 July 2007 - Revised: 18 October 2007 - Accepted: 22 October 2007 - Published: 12 November 2007

\begin{abstract}
A stacking method to detect very weak signals is introduced in this paper. This method is to stack observed data in different well known periodicities according to the astronomical clock since majority geophysical observations are time based. We validated this method by applying it in four different cases. Interactions behind the observed parameters become obviously after it is stacked in two diurnal and semidiurnal tidal periodical waves. Amplitude and phase variations will be also measurable when a sliding windows stacking is used. This could be an important reference to find precursors before some earthquakes and volcanic events, corresponding to attenuations of medium patterns.
\end{abstract}

\section{Introduction}

The new development of HiCum stacking method (Histogram Cumulating) is introduced in this paper (van Ruymbeke et al., 2003). Detect different periodical signals is an important issue in nature science, especially in the geophysical domain. Periodicities of signals have been widely studied since Fourier transform algorithm was published. However, in our study, we are more focused on the properties of different signals inside the pre-defined periodicities. Earth is continuously moving inside the gravitational gradient induced by the Moon and Sun. The orbital parameters of the motion are strictly defined by the celestial mechanics so that the reaction of the Earth to each outside body produces many periodical signals and most of them could be separated using stacking method if long enough time series are available. For instance, the lunar and solar semidiurnal wave could be separated with one month data. But, the ultimate purpose of the method is not only to separate the period signal of observed parameters, but also to detect the signal's amplitude and phase variations

Correspondence to: P. Zhu

(zhooping@gmail.be) in time domain and to connect them with past events such as earthquakes and volcanic events. For example, small variations of amplitude distribution before a strong earthquake have been found when it is used to investigate the relation between tidal stress and Vrancea seimic zone (Cadicheanu et al., $2007^{1}$ ).

Multi-parameters approach is required for the monitoring of complex interactions induced by tectonic activities in seismic, volcanic, geochemical areas, hydrology, land-slide zones. To illustrate local inter-activity between parameters, we analyzed four situations with HiCum, The barometric pressure induced on tidal gravity field variations in Membach (Belgium), sensor recording sea level in a lava tube in Lanzarote (Spain), monitoring of strain, pressure and temperature in underground cave at Rochefort (Belgium), and ground water level inside boreholes plunging in three aquifers at Uccle (Belgium). All of the examples show how the method works on each geophysical experiment.

\section{Description of "HiCum" stacking method}

Stacking data has been used for many decades in the meteorological fields (Bartels, 1938). In fact, as early as 19th century Darwin firstly used similar method to observe the sea tides cycle. Following that idea, Doodson further promoted the work and built the precisely sea tides forecast "DoodsonLégé" machine, which was in daily use since 1948 until it was superseded by the electronic computer during 1960s. The stacking method introduced here is based on similar principle; it separates observed signals according to predefined periodicities. Time based observation is a combination of periodical signals with nonlinear factors, so that main frequencies of the contained signal could be localized by the

\footnotetext{
${ }^{1}$ Cadicheanu, N., van Ruymbeke, M., and Zhu, P.: Tidal Triggering evidence of intermediate depth earthquakes in the Vrancea zone (Romania), Nat. Hazards Earth Syst. Sci., in review, 2007.
}

Published by Copernicus Publications on behalf of the European Geosciences Union. 

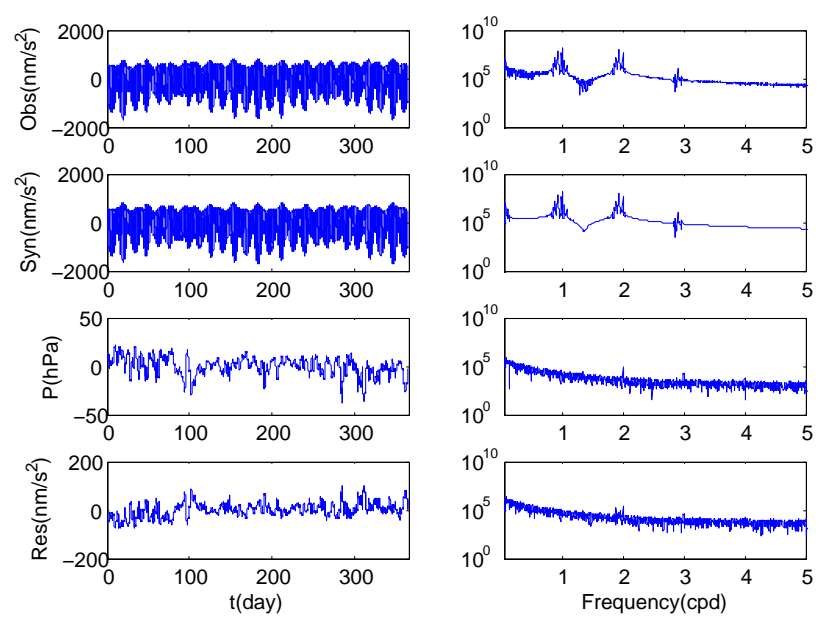

Fig. 1. (a) 365 days SG data Membach (Belgium), Synthetic tides, Gravity residuals, Barometric Pressure and (b) their corresponding Fourier spectrum.

Fourier Transform, an important hypothesis for the HiCum method being that the period is precisely priori defined. A periodical signal could be expressed as:

$f(t)=\frac{1}{N s} \sum_{i=1}^{N s} \sum_{j=1}^{T} y\left(t_{j}\right)+\varepsilon$

$i=1, T, 2 T, \ldots N s \quad j=1,2,3, \ldots . T$

$T=\frac{2 \pi}{\omega}$

where $f(t)$ represents averaging stacking results, $t$ the time, $y(t)$ the observed data, $N s$ the stacking times, $T$ the period, $\omega$ : the angular speed and $\varepsilon$ the uncertainties and errors. The precision of the stacking results is closely related with the data length $n$, the stacking period $T$, and the sampling interval $d t$. Then stacking results can be fitted by a sinusoidal function.

$s(t)=A \sin \left(\omega t_{j}+\alpha\right)$

where $\mathrm{A}$ is the amplitude and $\alpha$ the phase

$$
\begin{aligned}
& A=\sqrt{\sum_{j=1}^{T}\left(\left(f\left(t_{j}\right) \sin \left(\omega t_{j}\right)\right)^{2}+\left(f\left(t_{j}\right) \cos \left(\omega t_{j}\right)\right)^{2}\right)} \\
& \alpha=\tan ^{-1}\left(\frac{\sum_{j=1}^{T} f\left(t_{j}\right) \sin \left(\omega t_{j}\right)}{\sum_{j=1}^{T} f\left(t_{j}\right) \cos \left(\omega t_{j}\right)}\right)
\end{aligned}
$$
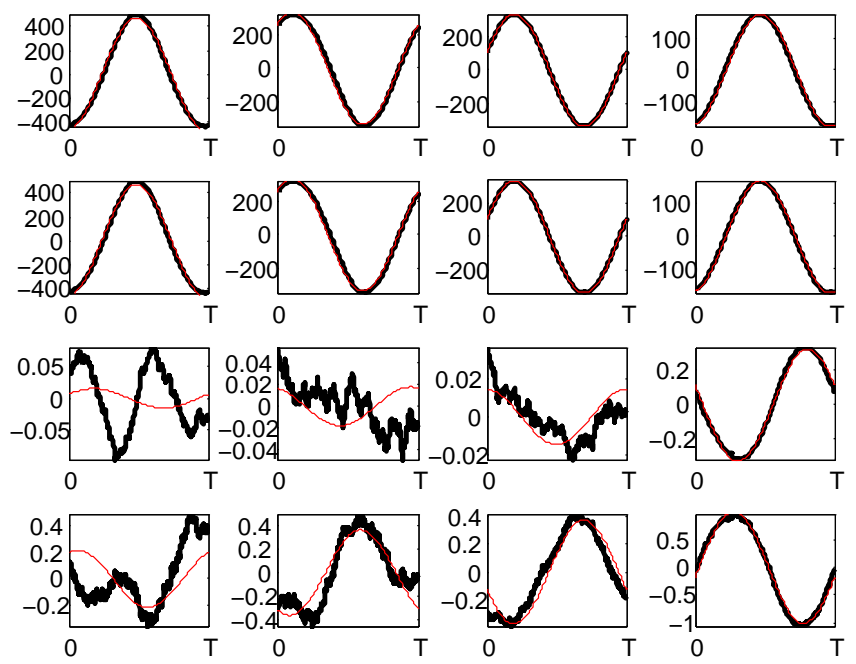

Fig. 2. HiCum Staking results of K1, O1, M2, S2 periods applied to the four channels which are observed tides, synthetic tides, gravity residuals and barometric pressure.

The goodness of the fit could be valued by root mean square error RMS

$\mathrm{RMS}=\sqrt{\frac{1}{T} \sum_{j=1}^{T}\left(f\left(t_{j}\right)-s\left(t_{j}\right)\right)^{2}}$

For instance, if one year gravity records with minute sampling is stacked in the lunar period M2 (central frequency 1.9504 cycle per day, corresponding to $745 \mathrm{~min}$ the period), the M2 wave could be separated by 705 (365.15 day*1440 $\mathrm{min} / 745 \mathrm{~min}$ ) times stacking and the mean square error will be reduced about $\sqrt{N s}$ times (this example is $\sqrt{705}=26$ times). Sometimes, for a high quality data bank with high sampling rate compare to the signal's period, the signal could be easily separated by several stacks. It provides a possibility to check the amplitude and phase variation for the signal in an approximated time interval. It is an important approach for the stacking method because, in the tidal triggering evidence of earthquakes and volcanic events studies, the amplitude and phase distribution changes in the tidal period could be a possible precursor for the forecasting of these events (Tanaka et al., 2006; Nicoleta et al., 2007). So that, the static stacking function is developed into a dynamic one by a sliding windowed stacking function:

$f_{w}(t)=\sum_{k=1}^{N} f(t)$

$f_{w}(t)=\sum_{k=1}^{N}\left[\frac{1}{N s} \sum_{i=1}^{N s} \sum_{j=1}^{T} y\left(t_{j}\right)+\varepsilon\right] \quad k=1, w, 2 w, 3 w, \ldots N$

where $f_{w}(t)$ is a windowed stacking result, $N$ the number of the windows, $w$ the sliding window length and $w \geq T$. 
Table 1. Selected astronomical periodicities and corresponding gravitational tides amplitudes for Belgium.

\begin{tabular}{lccc}
\hline Symbol & $\begin{array}{c}\text { Period } \\
\text { T(Min) }\end{array}$ & $\begin{array}{c}\text { Amplitude } \\
\left(\mathrm{nm} / \mathrm{s}^{2}\right)\end{array}$ & Origin \\
\hline K1 & 1436 & 431.2255 & Lunar \& Solar declinational wave \\
O1 & 1549 & 306.6215 & Lunar principal wave \\
M2 & 745 & 314.8163 & Lunar principal wave \\
S2 & 720 & 146.4691 & Solar principal wave \\
\hline
\end{tabular}

Through Eqs. (1), (3), (6), we can build up a matrix $\mathbf{E}$ with the stacking times $N s$ and its corresponding stacking results errors RMS

$\mathbf{E}=\left(\begin{array}{c}N s \\ \mathrm{RMS}\end{array}\right)$

The minimum stacking times $N s$ could be determined by optimizing the RMS value. So a long series record could be divided into $N$ continuous sliding windowed stacking, once the stacking time $N s$ fixed. Sliding stacking results give the amplitude and phase variation at approximated time interval $w$.

\section{Membach superconducting gravimeter record (SGs)}

We firstly selected a one year record of the Superconducting Gravimeter installed in the Membach station (Belgium). This station $\left(50.6092^{\circ} \mathrm{N}, 6.0067^{\circ} \mathrm{E}\right.$, altitude $\left.250 \mathrm{~m}\right)$ is among the best Belgian seismic stations and is usual as the reference point for the Belgian gravity network. It consists of a $130 \mathrm{~m}$ long gallery excavated in low-porosity argillaceous sandstone with quartzite beds.

We compared separately results of Fourier spectrum and HiCum stacking on 365 days data of theoretical tides, gravity residuals and barometric pressure (Fig. 1a). The synthetic tides was calculated by Tsoft with a local mode adjusted by long term SGs observations (Van Camp and Vauterin, 2005). The gravity residuals are directly computed by subtraction of the theoretical value from the observed tide. Diurnal, semidiurnal and ter-diurnal energies appear obviously in the spectrum of observed and synthetic tides. There are very small peaks in the semi-diurnal and ter-diurnal frequency band of the residuals and barometric Fourier spectrum (Fig. 1b.) by which it's difficult to figure out the barometric pressure effect on the SGs record. However, the pressure effect becomes very clear in the S2 period (Table 1) stacking results when the same data set is stacked in by HiCum. We selected the four waves which share $70 \%$ total energy of gravity tides coming from lunar and solar attraction (Table 1). K1 is the strongest wave and the barometric and ocean loadings have the minimum effect on O1 wave nearby Membach station (Melchior, 1978). Two main semidiurnal waves M2 and S2 are analyzed too (Fig. 2). The linear regression between the
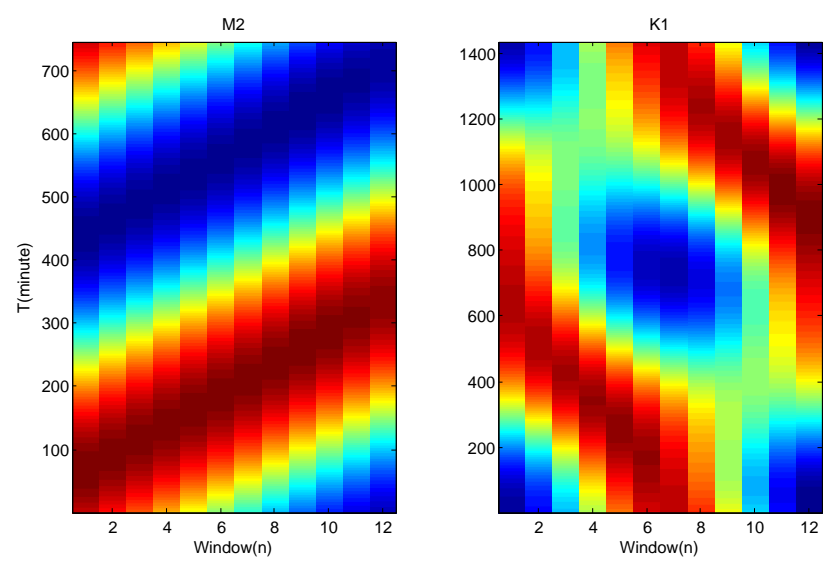

Fig. 3. Sliding windows stacking on $\mathrm{K} 1$ and $\mathrm{M} 2$ period, the beat effect from gravitational force appeared in $\mathrm{K} 1$, and lunar node effect could be observed in M2.

HiCum stacking results on four periods, confirms the quality of the synthetic mode adjustment with differences to the ideal unity $\left(1 \mathrm{~nm} / \mathrm{s}^{2}\right)$ factor smaller than $0.1 \%$ except for $\mathrm{S} 2$ which could be more perturbed by the main tidal component existing in the barometric spectrum. We compare the atmospheric pressure record and HiCum stacking residuals. Correlation appears clearly for the $\mathrm{K} 1$ and $\mathrm{S} 2$ component. There is very small barometric effect on M2 but it is well correlated with the residuals, the slope of best fit $\mathrm{S} 2$ wave is $2.829950 \mathrm{~nm} / \mathrm{s}^{2} / \mathrm{hPa}$ which is very near the global mode $\left(3.0 \mathrm{~nm} / \mathrm{s}^{2} / \mathrm{hPa}\right)$ for correction barometric pressure on $\mathrm{SGs}$ record (Table 2).

The sliding approach is applied on the K1 and M2 period. One year SGs records are stacked in a 30 days window and the window slided with 30 days step. For each window, we applied sliding stacking for the $\mathrm{K} 1$ and M2 periodicities (Fig. 3). The sequenced outputs show the change of the two periodicity waves in time domain. For M2 which is the main lunar principle wave, its amplitude should be nearly constant (Melchior, 1978). The 30 days windows allow separating the M2 component of the solar S2 one. The lunar orbit node is about 27.32 days which appears in the sliding stacking as a phase shift. The K1 period is totally different because it contains the action of solar and lunar declinations, so that appears the beat effect inside the K1 periodicity (Fig. 3). 
Table 2. Linear regressions between observed and synthetic tides, between residuals and barometric pressure.

\begin{tabular}{ccrccrrrrr}
\hline & \multicolumn{3}{c}{$\begin{array}{c}\text { Linear regression between observed } \\
\text { tides and synthetic tides }(y=a x+b)\end{array}$} & \multicolumn{4}{c}{$\begin{array}{c}\text { Linear regression between residuals } \\
\text { and barometic pressure }(y=a x+b)\end{array}$} \\
\hline & $\mathrm{a}$ & $\mathrm{b}$ & $R^{2}$ & $\mathrm{rms}$ & $\mathrm{a}$ & $\mathrm{b}$ & $R^{2}$ & $\mathrm{rms}$ \\
\hline $\mathrm{K} 1$ & 1.0003 & -0.000628 & 0.999998 & 0.467 & -2.756 & -0.000866 & 0.895025 & 0.155 \\
$\mathrm{O} 1$ & 0.9991 & 0.009490 & 1.000000 & 0.019 & -3.215 & 0.006789 & 0.116549 & 0.224 \\
$\mathrm{M} 2$ & 0.9992 & 0.003947 & 1.000000 & 0.131 & -6.633 & -0.004588 & 0.930038 & 0.063 \\
$\mathrm{~S} 2$ & 1.0017 & 0.000701 & 0.999971 & 0.668 & -2.830 & 0.000100 & 0.987746 & 0.077 \\
\hline
\end{tabular}
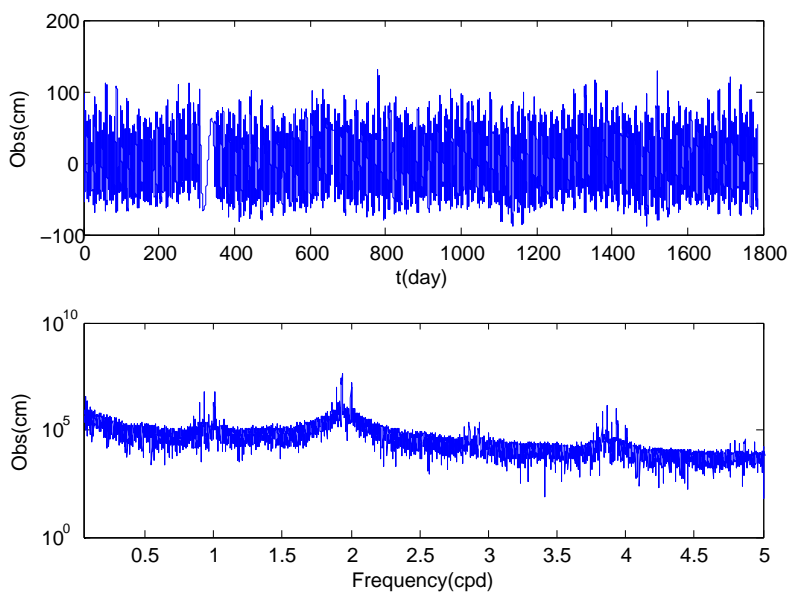

Fig. 4. (a), Jameos del Agua Sea-level sensor record on 5 years interval and (b) its Fourier Spectrum which confirms the non-linearity pattern with existing peaks between the fundamental diurnal periods until the fifth harmonic.

We can conclude from this example: the goodness of the synthetic tides mode and the barometric effect on gravimeter could be found after staking in four periods and the lunar orbit node effect are obviously in the sliding stack results.

\section{Lanzarote sea gauge}

The next example is an EDAS sea-level monitoring station ,set-up at Lanzarote Island (Spain) which is the most northeastern island of the Canarian Archipelago (van Ruymbeke, 2001). The landscape of Lanzarote is dominated by numerous volcanoes. The site of observation named "Jameos del Agua" is located in a lava tunnel of the quaternary volcano La Corona $(609 \mathrm{~m})$. The geometry of this tunnel consists in a series of small lakes interconnected by passages for water. This tunnel is plunging into the sea for more than one and half kilometers. Oceanic tidal there could reach few meters. The level depends continuously of the water flow in the tunnel which is function of the passage section. This is a typical situation of non linearity with transfer function influenced by the signal itself.
A sea gauge sensor records the sea level in the first open lake of the lava tunnel. Very smooth motion appears due to the filtering of short period sea waves through the few openings existing between the open-sea and the tunnel. Fourier spectrum confirms the nonlinearity of the water motion inside the lava tunnel (Fig. 4b).

The HiCum is applied to five years records. M2 amplitude is $40.528 \mathrm{~cm}$ of sinusoidal fit with RMS error 0.8261 and S2 amplitude $4.972 \mathrm{~cm}$ of sinusoidal fit with RMS error 0.2132 . It shows the efficiency of stacking to separate harmonic components on long registrations (Fig. 5).

\section{Rochefort strain meter}

This example concerns the detection of very weak signals (VWS) in a nosing signal of a strain meter. The instrument is located in the Rochefort karstic cave built-up by the erosion of the Lomme River (Belgium). The monitoring is assumed with several EDAS sensors. We apply the HiCum analysis on a file covering 800 day's records with four channels. The first channel is a glass stain meter RA1 located on a vertical crack. The second and the third channels are two thermometers monitoring temperature of the surface rock and environment in the gallery nearby the strain-meter. The fourth channel is a barometer (Fig. 6a) (van Ruymbeke et al., 2004).

The spectral analysis of the four channels shows different peaks in diurnal, semi-diurnal and ter-diurnal frequency bands (Fig. 6b).

For the strainmeter, diurnal and semi-diurnal activities appear clearly without harmonics. Contrarily for rock temperature and barometric pressure, semi-diurnal and ter-diurnal peaks exist and seem to be correlated. We subtract the raw dada with a third degree polynomial function to eliminate long term instrument drift before stacking the data (Fig. 7).

The length of strain meter is only $15 \mathrm{~cm}$. We observe that the HiCum for the strainmeter on M2 and S2 are very well determined. The amplitudes reach about 20 nanometers with a noise level of only a few nanometers! On the 4th channel, HiCum confirms a significant S2 barometric component with a negligible $\mathrm{M} 2$ one. The air thermometer HiCum on $\mathrm{S} 2$ is like the derivative of the barometric modulation, confirming 

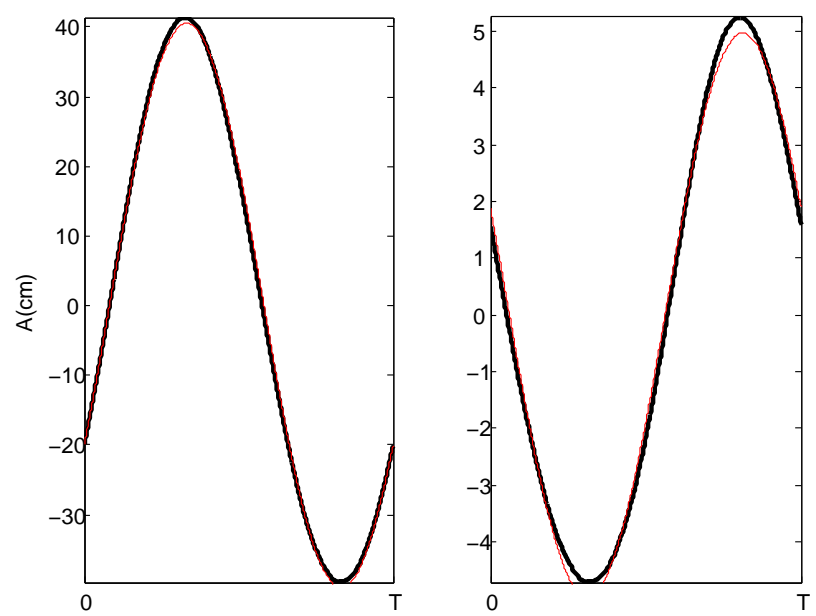

Fig. 5. HiCum of Jameos J31 sea gauge. HiCum results on M2 and $\mathrm{S} 2$ periods.
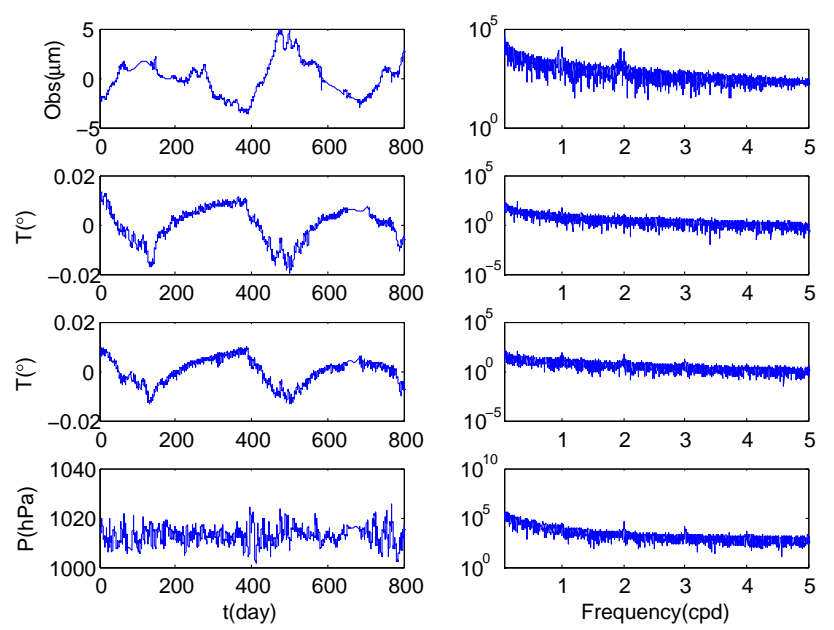

Fig. 6. (a) Four channels recording signals from strain-meter, air thermometer, rock thermometer and barometer. (b) Corresponding Fourier spectrum.

the induction process found with spectral analysis (Rasson and van Ruymbeke, 1977).

No significant effect appears for M2. For rock thermometer, only residual effects exist which could be related to evaporation of water flowing through cracks which could influence the strain-meter record.

These graphs illustrate the interests of the HiCum stacking method for problems with complex interactions. The records of strain-meter are very noisy with a lot of irregularities. However, HiCum on long time interval permits to extract clear information with sufficiently large dynamics to validate physics models.
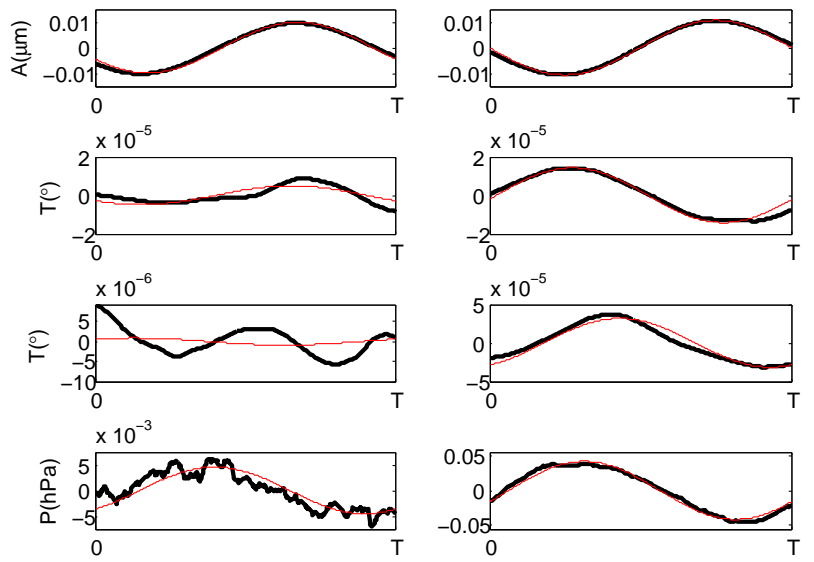

Fig. 7. HiCum applied to the raw data of four channels (strainmeter, rock thermometer, air thermometer and barometer) for lunar M2 period and solar S2 period.
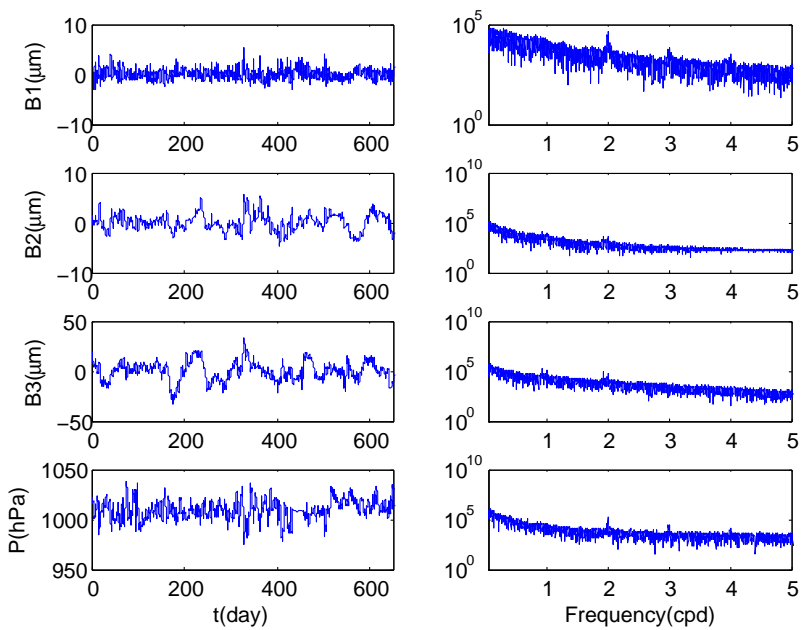

Fig. 8. (a) The four channels show the signals of the three boreholes water levels and, barometric pressure. (b) the corresponding Fourier spectrum.

\section{Uccl boreholes water levels}

We illustrate a medium interaction of actions by analyzing a borehole ground water level station located at the Royal Observatory of Belgium. It records the change of the water levels in three tubes plunging separately in three independent aquifers located at different depths (B1: $-35 \mathrm{~m}, \mathrm{~B} 2:-64 \mathrm{~m}$, B3: $-95 \mathrm{~m})$. The upper aquifer is an opened one, the second a semi-confined, and the third a confined one (Fig. 8a) (van Ruymbeke and Delcourt, 1985). The original records were pre-treated with the same procedure as the previously studied Rochefort one. Main conclusions from the spectral analysis concern interaction of water levels with simultaneous atmospheric pressure and tidal gravity changes. However the signal to noise ratio for spectrums is too low for understanding the relations between these two effects (Fig. 8b). 

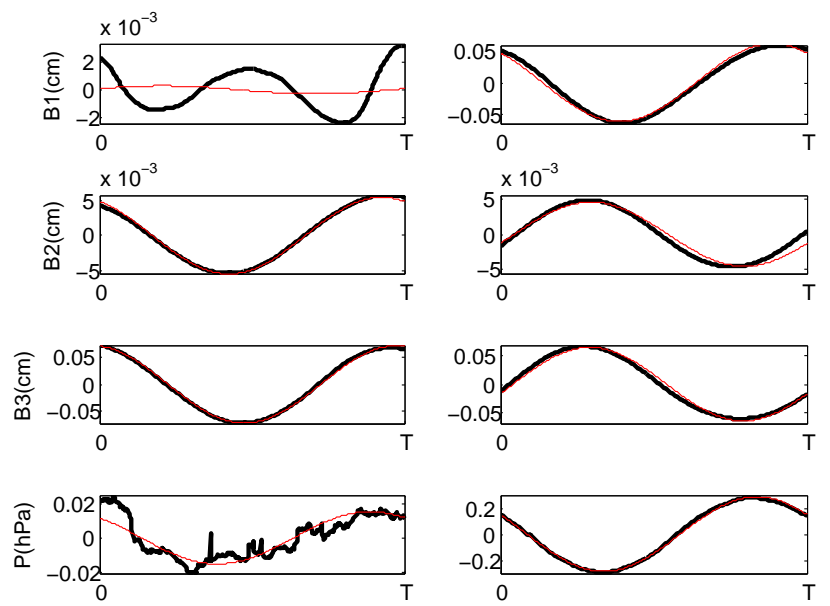

Fig. 9. HiCum with lunar M2 and solar S2 period graphs applied to the four channels (water levels B1, B2 \& B3 and barometer).

We apply the HiCum method on same series of data. For the first borehole and the barometric channel, HiCum shows a common large amplitude S2 and negligible modulations on M2. The gravitational effect appears on the B3-aquifer and smoothly on B1. Induction of atmospheric pressure looks negligible for the B2 and B3 boreholes. It is another example of this method to compare by stacking, two kinds of actions by the influences detected in reactions (Fig. 9).

\section{Discussion}

This paper has introduced a stacking method (HiCum) which could transform time based observation into different periodical based signals by stacking. One important issue in Earth science is to find periodical phenomena and to explain the physical meanings behind it. HiCum stacking method is another way to precisely study the performance of different signals in the frame of a known period $T$. The synthetic tide mode accuracy and barometric effect on gravimeter could be determined when the signals are stacked in four periods. Sliding stacking shows the well knows lunar node effect in the form of phase shift inside each window. In the sea gauge observations, the main semidiurnal lunar and solar tides signals could be subtracted and separated; for the strain meter records, very small tidal inductions can be detected even if the record is largely polluted by moisture, pressure and temperature. In the borehole water level monitoring stations, the semidiurnal tides signals are detected in the two deepest aquifers. Above all, these examples are just illustrating how the stacking method works. However, several aspects of the method still need to be investigated specially: its performance on short period signal studies and its adaptabilities in other domains.
Acknowledgements. The authors are grateful to all people who participate in EDAS projects; we thank particularly M. van Camp and M. Hendrickx for giving the Membach SG data, and E. de Kerchove correcting manuscript. The experiments in Lanzarote were organized with the support of R. Vieira and his colleagues; Local facilities are purchased by the Casa de los Volcanoes belongs to the Cabildo Insular de Lanzarote. G. Tuts has prepared the data files. The project in Rochefort was supported by Y. Quinif and Th. Camelbeeck. This research is financially supported in part by the Action 2 contract from the Belgian Ministry of Scientific Politics. We deeply appreciate the comments from B. Meurers and anonymous reviewer that served improves significantly the original manuscript.

Edited by: P. F. Biagi

Reviewed by: B. Meurers and another anonymous referee

\section{References}

Bartels, J. : Random Fluctuations, Persistence and Quasipersistence in Geophysical and Cosmical Periodicities, Terr. Magn. Atmos. Electricity, 40, 1, 1-60, 1938.

Melchior, P.: The Tides of the Planet Earth, Pergamon Press, London, 1978.

Rasson, J. and van Ruymbeke, M.: Underground meteorology at the underground Laboratory of Geodynamics at Walverdange, in: VIIIth Symp. on Earth Tides, Bonn, 1977.

Tanaka, S., Sato, H., Matsumura, S., and Ohtake, M.: Tidal triggering of earthquakes in the subducting Philippine Sea plate beneath the locked zone of the plate interface in the Tokai region, Japan, Tectonophysics, 417, 69-80, 2006.

van Camp, M. and Vauterin, P.: Tsoft: graphical and interactive software for the analysis of time series and Earth tides, Comput. Geosci., 31(5) 631-640, 2005.

van Ruymbeke, M., Howard, R., Pütz, P., Beauducel, F., Somerhausen, A. and Barriot, J-P.: An Introduction to the use of HiCum for signal analysis, BIM 138, 10 955-10 966, 2003.

van Ruymbeke, M.: Projects of the Royal Observatory of Belgium (ROB) at the Lanzarote Geodynamical Laboratory (LGL), J. Geodetic Society of Japan, 47, 463-469, 2001.

van Ruymbeke, M. : The Environmental Data Acquisition System(EDAS) developed at the Royal Observatory of Belgium, J. Geodetic Society of Japan, 47, 40-46, 2001.

van Ruymbeke, M. and M. Delcourt : A capacitive transducer for water level,International Symposium on Earth Tides, Consejo Superior de Investigaciones Cientificas, Madrid, 95-103, 1985.

van Ruymbeke, M., Beauducel, M. Fr., and Somerhausen, A.: The Environmental Data Acquisition System(EDAS). Developed at the Royal Observatory of Belgium, in: Short thermal and hydrological signatures related to tectonic activity, Cahier du Centre European de Geodynamique et de Seimologie, 14, 163-174, 1997.

van Ruymbeke, M., Shaoming, L., Quinif, Y., and Camelbeeck, T.: The Monitoring of Tectonic Movements In Nature Caves, the 3rd International Conference on Continental Earthquakes Mechanism, Prediction, Emergency Management and Insurance, Beijing, 134-140, 2004. 